\title{
Editorial overview: Chemical genetics and epigenetics
}

\section{Danica Galnić Fujimori and Stuart J. Conway}

This issue of the Current Opinions in Chemical Biology highlights recent advances in the understanding of epigenetic processes. Epigenetic modifications - both to histone proteins and associated DNA - encompass a growing number of chemical modifications to chromatin, which often have profound regulatory roles in diverse cellular processes. Misregulation of these modifications is associated with a number of diseases, promoting the development of chemical probes and therapeutics that target protein families involved in deposition, recognition, and removal of epigenetic marks. Recent advances in mapping of RNA modifications have led to identification of a number of modified nucleotides and birth of an emerging field of RNA epigenetics. This issue highlights advances in understanding cellular function, regulation, and inheritance of these modifications. In addition, development of chemical tool compounds to probe and perturb the function of proteins involved in writing, reading and erasing of epigenetic marks is also discussed. Application of chemical genetic approaches to interrogate epigenetic enzymes, as well as development of advanced analytical platforms to quantitatively assess modification status are also covered.

Several reviews in this issue address development of chemical probes for epigenetic protein families, and their use in pharmacological validation of these proteins as potential therapeutic targets. Over the past few years, development of small molecule inhibitors for bromodomains has attracted significant attention both in academia in industry, resulting in more than a dozen inhibitors currently in clinical trials. These efforts have largely been focused on bromodomain and extra terminal (BET) family of bromodomains. In this issue, Runcie et al. have reviewed the innovative application of chemical genetics to epigenetic targets, with a focus on the use of "bump and hole" methodology to gain selectivity between the BET bromodomain family members. They also discuss the application of proteolysis-targeting chimeras (PROTACS) to the BET bromodomains. Theodoluou et al. highlight advances in development of chemical probes for the broader family of non-BET bromodomains and their potential to treat human diseases. Histone or lysine deacetylases (HDACs/KDACs) are one of the more established classes of epigenetic drugs. Leus et al. have reviewed the emerging role of HDAC3 in NF-kB-mediated inflammation. Protein arginine deiminases (PAD), an enzyme class that converts arginine to citrulline, are review by Lewis and Nacht. The authors provide an overview of the involvement of these enzymes in pathology of several diseases, and highlight therapeutic potential of PAD inhibitors in disease models. Schapira and Arrowsmith discuss recent progress in development of methyltransferase inhibitors, discussing our current understanding of their their diverse modes of actions, and their application in oncology. In addition, the authors summarize recent insights into clinical efficacy of DNA methyltransferase inhibitors which support a combination of epigenetic drugs and immunotherapy as a potential novel approach to treat cancer. Focusing on methylation erasers, Nowak et al. highlight advances in understanding catalytic mechanisms and critical structural features of histone demethylases. Progress towards development of subfamilies specific chemical probes and for metabolic regulation of this class of epigenetic proteins is also discussed. James and Frye have summarized the relatively recent progress being made in the development of chemical probes for methyl-lysine reader domains. The authors have provided an overview of both small molecule and peptide ligands, with a particular interest in chromodomains. Hardivillé and Hart have summarized progress in our understanding of the O-GlcNAcylation post- 
translational modification (PTM), which is linked to nutrient availability. The authors provide a fascinating insight into the link that this PTM provides between nutrient availability and epigenetic effects in diseases including diabetes and cancer. Liu et al. have focused on modifications on DNA, rather than histone proteins. They have focused on the increasing number of cytosine modifications that have been identified, and looked at the effect caused by these DNA marks.

Development of analytical platforms to analyze complexity of chromatin modifications is of critical importance to understanding co-occupancy and changes in response to perturbations (e.g. drugs). Capitalizing on advances in mass spectrometry, Zheng et al. provide an insight into strategies for quantitative analysis of histone modifications. Like DNA, RNA is also a subject to modifications that are introduced post-transcriptionally and have profound impact on function. Li et al. describe progress in transcriptome-wide detection of pseudouridine in RNA, as well as biogenesis and known functions of this modification.

Blake and Watson consider one of the key questions in the area, which is how the epigenetic effects caused by environmental factors are transgenerationally inherited. Following epigenetic information over multiple generations is highlighted as a significant challenge, but one which is essential to understand for the progress of the field.

By providing summary of the current status in the field and outlining outstanding challenges, we hope that this issue attracts attention from both current researcher and highlight opportunities to those interested in entering the field of chemical epigenetics. We would like to thank the authors for their outstanding contributions to the special issue. We also thank the reviewers of manuscripts for this issue who have provided careful and insightful reviews of the submissions. Particular thanks go to the editorial office staff for their support of this issue.

\section{Danica Galnić Fujimori}

Danica Galonić Fujimori obtained her B.Sc. in from Belgrade University, and her PhD in organic chemistry at University of Illinois at Urbana-Champaign, where she trained with David Gin and Wilfred van der Donk. Following postdoctoral training with Chris Walsh at Harvard Medical School, she joined the faculty in Cellular and Molecular Pharmacology and Pharmaceutical Chemistry Departments at University of California San Francisco, where she is an Associate Professor.

\section{Stuart J. Conway}

Stuart Conway is a Professor of Organic Chemistry at the University of Oxford and the Fellow in Organic Chemistry at St Hugh's College, Oxford. After completing a PhD at the University of Bristol, he undertook post-doctoral studies at the University of Cambridge with Andrew Holmes. Between 2003 and 2008 he was a Lecturer in Bioorganic Chemistry at the University of St Andrews. His research focuses on the use of chemical techniques to interrogate biological questions in a range of areas, including epigenetics. 